\title{
Aplicação de métodos determinísticos para a obtenção de gap crítico em rotatórias urbanas brasileiras
}

\author{
Diego Fernandes Neris ${ }^{1}$, Antonio Clóvis Pinto Ferraz ${ }^{2}$, Ana Paula Camargo Larocca ${ }^{3}$ \\ ${ }^{1}$ Departamento de Engenharia de Transportes, Escola de Engenharia de São Carlos, USP, diego.neris@usp.br \\ ${ }^{2}$ Departamento de Engenharia de Transportes, Escola de Engenharia de São Carlos, USP, coca@sc.usp.br \\ ${ }^{3}$ Departamento de Engenharia de Transportes, Escola de Engenharia de São Carlos, USP, larocca.ana@usp.br
}

\section{Recebido:}

10 de outubro de 2018

Aceito para publicação:

05 de maio de 2019

Publicado:

31 de dezembro de 2019

Editor de área:

Sara Ferreira

Palavras-chaves:

Rotatória,

Gap crítico,

Capacidade.

\section{Keywords:}

Roundabout,

Critical gap,

Capacity.

DOI:10.14295/transportes.v27i4.1866

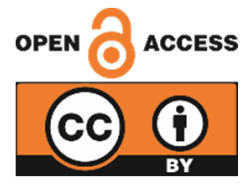

\begin{abstract}
RESUMO
O presente estudo tem o objetivo de avaliar o gap crítico e o follow-up headway para rotatórias brasileiras e comparar com estudos estrangeiros. Para tanto, foram selecionadas três rotatórias brasileiras de duas faixas de circulação e de entrada. Para a obtenção de gaps críticos foram utilizados cinco métodos determinísticos: Siegloch (1973), Raff (1950), Miller \& Pretty (1968), Wu (2012) e Bunker (2012), sendo que pelo primeiro método foi possível obter também o follow-up headway. Os valores de gaps críticos foram consideravelmente diferentes em função da faixa utilizada: na faixa da direita, os motoristas aceitam gaps menores para entrar na interseção. Esta diferença não foi significativa quando se trata de valores de follow-up headway. Em seguida, os métodos de estimação de gap crítico foram validados comparando a capacidade das interseções pelo método de Hagring (1998) com dados de fluxos agregados por minuto. Finalmente, os valores brasileiros foram comparados com dados estrangeiros e foram verificadas semelhanças com países como Suíça, Itália e Portugal.
\end{abstract}

\begin{abstract}
This paper focuses on evaluating the critical gap and the follow-up headway for Brazilian roundabouts and on comparing it to the results of foreign studies. Three two-lane roundabouts were selected to obtain the critical gap through five methods: Siegloch (1973), Raff (1950), Miller \& Pretty (1968), Wu (2012) and Bunker (2012). Through the first method, it was also possible to obtain the follow-up headway. The critical gap values were considerably different in each lane: on the right lane, the drivers accepted smaller gaps to cross to the intersection. This difference was not significant when it came to the follow-up headway. After that, the critical gap estimation methods were validated by comparing the intersection's capacity using the Hagring (1998) method with the combined data per minute of traffic flow. Finally, the resulting Brazilian values were compared to foreign data. There were similarities to the results found in countries like Switzerland, Italy and Portugal.
\end{abstract}

\section{INTRODUÇÃO}

O sistema viário urbano deve ser adequado para conduzir o tráfego de veículos e pedestres com segurança e fluidez. Essas características são muito importantes, principalmente nos cruzamentos, uma vez que esses constituem pontos críticos do sistema viário no tocante à capacidade do tráfego e acidentes. Quando o fluxo de veículos em uma interseção é pequeno, a operação com a via mais movimentada tendo preferência de passagem é a solução normalmente empregada. No entanto, quando o volume de veículos é maior, outras soluções devem ser equacionadas, tais como semáforo, rotatória ou viaduto (transposição em desnível). 
A interseção do tipo rotatória oferece a equidade de hierarquia entre as aproximações, maior fluidez ao trânsito, reduz a necessidades de paradas, e facilita a conversão à esquerda, pois não há conflito com veículos de sentido oposto. Como resultado, os atrasos e filas são reduzidos (Federal Highway Administration, 2010).

A capacidade da rotatória e de qualquer outro tipo de interseção sem semáforos depende do processo de aceitação de gap, função cognitiva de julgamento sobre a possibilidade de entrada na via principal, neste caso a via circular, por parte dos motoristas das aproximações (que não possuem a referência). Rotatórias oferecem a simplicidade de uma interseção de três aproximações, no formato "T", para interseções com mais aproximações, além do fluxo na via principal ser em sentido único, facilitando assim os processos de tomada de decisão.

Dois parâmetros são essenciais para entender o processo de aceitação de gap e, consequentemente, entender a capacidade da rotatória. O primeiro é o gap crítico, ou seja, mínimo gap que um veículo, da aproximação não preferencial, aceita para entrar na via principal. Esse parâmetro varia em função da atitude do motorista, da dimensão da rotatória e pode variar em função do fluxo de veículos (Polus, 2005). 0 segundo parâmetro representa como esses gaps são aproveitados, ou seja, a quantidade de veículos que entra em um mesmo gap, calculado em função do headway médio entre esses veículos. Este parâmetro é chamado de follow-up headway (TRB, 2010).

\section{CONTEXTUALIZAÇÃO TEÓRICA}

O gap crítico não pode ser medido diretamente através de observação em campo, portanto existem técnicas determinísticas que podem estimá-lo a partir da distribuição de gaps na via preferencial e com a classificação de quais foram aceitos ou rejeitados. Entre as técnicas, destacam-se as três mais utilizadas: Siegloch (1973), Raff (1950) e Miller \& Pretty (1968). Existem ainda outras duas técnicas mais recentes que são as de Wu (2012) e de Bunker (2012) que foram utilizadas no trabalho de Vasconcelos (2014) para aplicação em Portugal.

Já o follow-up headway pode ser determinado por observação do headway médio da fila de veículos que entra na rotatória em um mesmo gap ou pode ser determinado pelo método de Siegloch (1973), o mesmo método que determina o gap crítico.

Hagring (2000) salienta que, independentemente do método utilizado para a determinação de gap crítico, existem duas situações que podem ser consideradas em casos de rotatórias de múltiplas faixas: a primeira ao realizar o levantamento de gaps por faixa da via circular e a segunda ao considerar como se fosse um fluxo apenas, portanto definindo gaps entre veículos, independente das faixas que estejam. Vasconcelos (2014) verificou que os resultados para o segundo caso são mais compatíveis com a realidade, pois mesmo que o veículo da aproximação decida entrar na faixa externa da rotatória, o fluxo na faixa interna também interfere no processo de aceitação de gap.

Segundo o Highway Capacity Manual 2010 (TRB, 2010), para estudos realizados nos EUA, o valor do gap crítico em rotatórias varia entre 4,1 e 4,6 segundos enquanto o valor de follow-up headway varia entre 2,6 e 3,1 segundos. Diversos autores realizaram o estudo de gap crítico e follow-up headway para rotatórias de duas faixas de circulação. A seguir são apresentadas as experiências para a Suécia, Suíça, EUA, Itália, Portugal e Índia.

Hagring et al. (2003) realizaram a análise de gap crítico para a Suécia em uma rotatória de duas faixas. Os autores identificaram uma leve queda no valor de gap crítico conforme o fluxo na via circular aumenta. $\mathrm{O}$ valor médio do gap crítico encontrado no estudo de Hagring et al. 
(2003) foi de 4,08 segundos para a faixa da direita e 4,36 segundos para a faixa da esquerda, enquanto o follow-up headway médio foi de 2,64 segundos.

Lindenmann (2006), em seu estudo aplicado na Suíça, afirma que os veículos da faixa da esquerda, por ter condições de visibilidade melhor, tendem a partir antes para entrar na rotatória. Em contrapartida, os veículos da faixa da direita trafegam com um follow-up headway menor. Lindenmann (2006) encontrou o valor de 3,49 segundos de gap crítico para a faixa da esquerda e 3,45 segundos para a faixa da direita e, ao analisar as duas faixas em conjunto, encontrou uma média de 3,65 segundos. 0 follow-up headway encontrado nos estudos da Suíça foi 2,73 segundos para a faixa da esquerda e 2,55 segundos para a faixa da direita.

Rodegerdts et al. (2007) realizaram a análise de gap crítico em rotatórias de múltiplas faixas dos EUA e determinaram um gap médio de 4,85 segundos para a faixa da esquerda e 4,15 para a faixa da direita. 0 follow-up headway foi 3,90 segundos para a faixa da esquerda e de 3,55 para a faixa da direita, valores consideravelmente superiores aos propostos pelo HCM 2010 (TRB, 2010).

Gazzarri et al. (2012) realizaram as análises de gaps críticos em rotatória de múltiplas faixas da Itália. Para a faixa da esquerda foi encontrado o valor de 3,76 segundos, enquanto para a faixa da direita, 3,60 segundos. 0 follow-up headway, determinado através de observação em campo, resultou em 2,65 segundos para a faixa da esquerda e 2,64 segundos para a faixa da direita, portanto praticamente não houve alteração.

Vasconcelos (2014) realizou o estudo de gap crítico e follow-up headway para rotatórias localizadas em Portugal. 0 gap crítico encontrado foi de 3,40 segundos para a faixa da esquerda e de 3,20 para a faixa da direita. 0 follow-up headway resultou em um valor médio de 2,2 segundos para as duas faixas de entrada.

Na Índia, Rao et al. (2016) realizaram estudos em seis rotatórias e obtiveram resultados discrepantes em comparação aos outros países. 0 gap crítico obtido foi de 1,87 segundos, sem distinção de faixas, pois não havia sinalização horizontal limitando-as, tanto nas aproximações quanto na via circular das rotatórias. Para o follow-up headway, o valor observado foi de 1,15 segundos, também muito inferior aos encontrados em outros países. Mahesh, Ahmad e Rastogi (2014), que realizaram estudos semelhantes na Índia, encontraram um gap crítico de 2,2 segundos e follow-up headway de 1,2 segundos. Os autores citam que os valores são discrepantes devido ao alto fluxo de veículos sob duas rodas (que se aproxima a $40 \%$ do fluxo total) e pelo comportamento mais agressivo dos motoristas, justificando que em países em desenvolvimento os valores tendem a ser menores.

A determinação do gap crítico e follow-up headway são fundamentais para a calibração de modelos de capacidade de interseção. 0 modelo proposto por Hagring (1998) possibilita a aplicação segregada por faixa de entrada na rotatória, conforme indicado na equação a seguir.

$$
Q_{k}=\frac{e^{\left[-\sum \lambda_{i}\left(t_{c, k}-\Delta_{i}\right)\right]} \times \sum \lambda_{i}}{1-e^{\left(-\Sigma \lambda_{i} t_{f, k}\right)}} \prod \frac{\phi_{i}}{\phi_{i}+\lambda_{i} \Delta_{i}}
$$

em que

$$
k: \quad \text { Índice da faixa de entrada (direita ou esquerda) }
$$

$\lambda_{i}$, $\phi_{i}$ e $\Delta_{i}$ : Parâmetros de Cowan para a faixa "i" da via prioritária (circular)

$t_{c, k}: \quad \quad$ Gap crítico da faixa de entrada "k" (s)

$t_{f, k}: \quad$ Follow-up headway da faixa de entrada "k" (s) 


\section{METODOLOGIA}

Foram selecionadas três rotatórias modernas (com preferência para a via circular), duas em Araraquara/SP e uma em Campinas/SP, com duas faixas de circulação cada. Foram realizadas gravações de uma hora, em cada rotatória, na aproximação com maior fluxo de veículos (para a obtenção de maior número de dados possível).

A primeira rotatória, localizada em Araraquara/SP (Avenida Abdo Dajn, Rua João Batista de Oliveira, Rua Genesia Schiavinato e Rua Piauí), é uma interseção com quatro aproximações cujo diâmetro externo é de, aproximadamente, 40 metros. A aproximação analisada possuiu o dobro de fluxo de veículos ao comparar com o fluxo conflitante (na via circular). A segunda rotatória, também em Araraquara (Avenida Presidente Vargas e Rua Castro Alves), possui um diâmetro externo aproximado de 54 metros e, diferentemente da primeira, o fluxo predominante foi na via circular. A última rotatória analisada encontra-se no município de Campinas (Avenida Doutor Ângelo Simões, Avenida Marechal Carmona e Rua Engenheiro Roberto Mange) e possui um diâmetro externo de aproximadamente 50 metros com o fluxo semelhante entre a aproximação analisada e a via circular.

A partir de um ponto de referência de passagem de veículos em cada vídeo, foram contabilizados e medidos todos os gaps na via circular com o auxílio da ferramenta Macro da Microsoft Excel para obter maior precisão na cronometragem e para identificar a quantidade de veículos que entra em cada gap. Em seguida, foram aplicadas as metodologias de estimação de gaps críticos apresentadas a seguir para a caracterização de cada interseção.

\subsection{Siegloch (1973)}

O método de Siegloch é o único dos métodos que determina além do gap crítico, o follow-up headway. A limitação desse método é que deve ser aplicado apenas em situações em que há filas na aproximação em análise, ou seja, situações de saturação, para que não haja distorções na determinação do follow-up headway. 0 método é aplicado da seguinte forma:

- Determina-se o gap médio necessário para cada quantidade de veículos entrando em fila na rotatória.

- Elabora-se o gráfico que relaciona a quantidade de veículos que entram em fila e o gap médio para que ocorra esta entrada.

- Identifica-se a equação linear de tendência do gráfico elaborado. Nesta equação, o termo independente é chamado de " $t_{0}$ " e o termo dependente representa o follow-up headway em segundos, identificado como " $t_{f}$ ".

- Aplica-se a equação a seguir para a determinação do gap crítico (em segundos) pelo método de Siegloch (1973), cujas variáveis foram identificadas na etapa anterior.

$$
t_{c}=t_{0}+\frac{t_{f}}{2}
$$

\subsection{Raff (1950)}

0 método de Raff retorna o valor mediano do gap crítico, porém é razoável para a utilização em modelos de capacidade e pode ser determinado conforme o procedimento a seguir:

- Identificam-se os gaps aceitos e rejeitados, separando-os e ordenando-os do menor para o maior valor. Dentre os gaps rejeitados pelo mesmo veículo, deve-se considerar apenas o maior, partindo do princípio que os motoristas não aceitariam gaps menores que 
aqueles rejeitados. Este fato pode não ocorrer já que o tamanho do gap esperado pelo motorista para entrar na via principal pode reduzir conforme aumenta o tempo de espera.

- Elaboram-se funções de distribuição acumuladas desses gaps, uma para os gaps aceitos - $\mathrm{Fa}(\mathrm{t})$ - e outra para os gaps rejeitados - Fr(t).

- Plotam-se os gráficos das funções acumuladas dos gaps aceitos e rejeitados, sendo que para este último, plota-se a função complementar, ou seja, 1-Fr(t).

- valor mediano do gap crítico é aquele relacionado à interseção das duas funções.

\subsection{Miller \& Pretty (1968)}

0 método de Miller \& Pretty, também chamado de método da máxima verossimilhança, realiza uma análise comparativa entre o maior gap rejeitado por um motorista e o gap aceito pelo mesmo motorista, portanto considera apenas os motoristas que rejeitaram ao menos um gap, reduzindo assim a quantidade da amostra. Além disso, devem ser eliminadas as situações em que o gap aceito é menor que o maior gap rejeitado. 0 método é aplicado da seguinte forma:

- Realiza-se a distribuição acumulada do tipo log-normal para cada gap aceito e maior gap rejeitado por cada motorista, conforme a equação a seguir:

$$
F(t)=\frac{1}{2}+\frac{1}{2} \operatorname{erf}\left(\frac{\ln x-\mu}{\sqrt{2} \sigma}\right)
$$

em que $\quad \mathrm{F}(\mathrm{t}): \quad$ Função log-normal podendo ser para o gap aceito - $\mathrm{Fa}(\mathrm{t})$ - ou para o gap rejeitado - Fr(t),

$\mathrm{x}: \quad$ gap a ser considerado (s)

$\operatorname{erf}(\mathrm{x}): \quad$ Função erro de " $\mathrm{x}$ "

$\mu$ e $\sigma: \quad$ Média e desvio-padrão, parâmetros a serem otimizados conforme as etapas a seguir.

- Para cada motorista "d", realiza-se o cálculo da diferença entre a função de distribuição log-normal do gap aceito $\left(a_{d}\right)$ e a função de distribuição log-normal do gap rejeitado $\left(r_{d}\right)$ e, ao final, determina-se o produto dessas diferenças para todos os " $\mathrm{n}$ " motoristas:

$$
L=\prod_{d=1}^{n}\left[F_{a}\left(a_{d}\right)-F_{r}\left(r_{d}\right)\right]
$$

- A equação anterior é de verossimilhança que deve ser maximizada, com o auxílio da ferramenta "Solver" do Excel ou similar, com a finalidade de obter o valor médio $(\mu)$ e o desvio-padrão $(\sigma)$ relativos ao produtório máximo.

- gap crítico $\left(t_{c}\right)$ pelo método de Miller \& Pretty é determinado pela equação a seguir.

$$
t_{c}=e^{\mu+\frac{1}{2} \sigma^{2}}
$$

\section{4. $\mathrm{Wu}(2012)$}

O processo para a determinação de gap crítico pelo método de $\mathrm{Wu}$, também chamado de equilíbrio das probabilidades, é mais recente e trabalha com os mesmos dados do método de Raff (1950), porém não é necessário separar os gaps aceitos dos rejeitados, conforme o processo a seguir:

- Devem-se ordenar os valores de gaps, do menor valor para o maior, independente se foi aceito ou rejeitado, porém identificando-os em coluna auxiliar.

- Aplica-se a função de distribuição acumulada de forma individual (colunas separadas) 
entre gaps aceitos e rejeitados. Quando um gap for classificado como aceito, por exemplo, atualiza-se a função acumulada de gaps aceitos e deve mantém-se o último valor da função acumulada de gaps rejeitados. 0 contrário também ocorre, mantendo assim um valor de função de distribuição acumulada para gap aceito ou rejeitado para todos os gaps (independentemente de sua classificação).

- Para cada gap, calcula-se uma estimativa da função densidade acumulada do gap crítico - $F_{t c}(t)$ - da seguinte forma:

$$
F_{t c}(t)=\frac{F_{a}(t)}{F_{a}(t)+1-F_{r}(t)}
$$

sendo que $\quad F_{a}(t): \quad$ Função de distribuição acumulada de gaps aceitos.

$F_{r}(t): \quad$ Função de distribuição acumulada de gaps rejeitados.

$t: \quad$ gap considerado (s).

- Em seguida, determina-se a frequência dos gaps críticos estimados através da diferença entre o valor da função densidade acumulada do gap crítico estimado atual $\left(t_{j}\right)$ e do anterior $\left(t_{j-1}\right)$ :

$$
p_{t c}\left(t_{j}\right)=F_{t c}\left(t_{j}\right)-F_{t c}\left(t_{j-1}\right)
$$

- Calcula-se a média do gap atual com o anterior e, finalmente, multiplica-se esse valor pela frequência dos gaps críticos estimados - $p_{t c}\left(t_{j}\right)$ - descrito na etapa anterior, obtendo uma multiplicação para cada gap.

- Finalmente devem-se somar todos os produtos citados na etapa anterior, sendo essa soma o valor do gap crítico pelo método de Wu (equilíbrio das possibilidades).

0 método de Wu realiza uma estimativa de uma função densidade acumulada do gap crítico baseado em cada gap determinado em campo. Este método atribui maior peso para os dados intermediários de gaps, minimizando possíveis erros causados por acontecimentos fora do padrão, além de ser resolvido apenas por cálculos simples, sem estimativas de parâmetros ou interpretações gráficas.

\subsection{Bunker (2012)}

No método de Bunker os dados a serem considerados são os mesmos do método de Miller \& Pretty (1968): o maior gap rejeitado e o gap aceito por cada motorista, ou seja, considera apenas motoristas que rejeitaram, ao menos, um gap. A determinação do gap crítico é realizada conforme o procedimento a seguir.

- Elabora-se uma lista de possíveis gaps críticos. No caso deste trabalho, variou-se de zero até 8 segundos em intervalos de 0,01 segundos.

- Para cada valor dos possíveis gaps $(\mathrm{t})$, deve-se somar a quantidade de intervalos de gaps (entre o gap rejeitado - $t_{r}$ - e o gap aceito $-t_{a}$ ) levantados em campo de cada motorista que os abrangem esses possíveis gaps, ou seja, todos os casos em que $t_{r}<\mathrm{t}<t_{a}$.

- Ao final, elabora-se o gráfico que relaciona o número de casos em que ocorreram a situação acima descrita $\left(t_{r}<\mathrm{t}<t_{a}\right)$ e os valores de possíveis gaps $(\mathrm{t})$, sendo considerado o gap crítico aquele que esteve incluso no máximo intervalos entre gaps rejeitados e aceitos por cada motorista. 


\section{RESULTADOS E DISCUSSÕES}

Inicialmente, foi observado em campo que há influência, na reação dos motoristas, da faixa em que o veículo se encontra para entrar na via principal: os veículos que se aproximam pela faixa da direita tendem a aguardar por um gap ultrapassando a faixa de retenção, já ocupando parte da rotatória. Sendo assim, é importante que a análise seja realizada por faixa de aproximação.

Na Tabela 1 são apresentados os resultados encontrados pelos métodos de determinação de gap crítico para cada uma das três rotatórias analisadas, separados por faixa de entrada e por método.

Tabela 1 - Gaps críticos (em segundos)

\begin{tabular}{|c|c|c|c|c|c|c|c|}
\hline \multirow{6}{*}{ 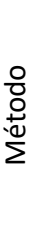 } & Rotatória & \multicolumn{2}{|c|}{ Araraquara 1} & \multicolumn{2}{|c|}{ Araraquara 2} & \multicolumn{2}{|c|}{ Campinas } \\
\hline & Faixa & Esquerda & Direita & Esquerda & Direita & Esquerda & Direita \\
\hline & Raff & 3,58 & 3,12 & 3,38 & 2,78 & 3,42 & 2,95 \\
\hline & Miller \& Pretty & 3,96 & 3,26 & 3,72 & 3,12 & 3,73 & 3,33 \\
\hline & $\mathrm{Wu}$ & 3,80 & 3,04 & 3,55 & 2,83 & 3,62 & 2,98 \\
\hline & Bunker & 3,72 & 3,02 & 3,53 & 3,29 & 3,48 & 3,12 \\
\hline
\end{tabular}

Para todos os casos analisados, o gap crítico resultou menor quando a faixa de entrada na rotatória é a da direita. A maior diferença verificada foi na rotatória "Araraquara 2" ao aplicar o método de Wu (2012), sendo 25\% maior o gap crítico na faixa da esquerda em relação ao da direita.

Em geral, os menores valores foram obtidos ao aplicar o método de Siegloch, pois é o método que utiliza apenas dados em situação de saturação, confirmando a influência do tempo de espera do veículo na determinação do gap crítico.

Pelo método de Siegloch ainda foi possível determinar o follow-up headway, que resultou entre 2,51 e 2,83 segundos, conforme a Tabela 2 a seguir.

Tabela 2 - Follow-up headway (em segundos)
\begin{tabular}{lll}
\hline \multirow{2}{*}{ Rotatória } & \multicolumn{2}{l}{ Faixa de entrada } \\
\cline { 2 - 3 } & Esquerda & Direita \\
\hline Araraquara 1 & 2,68 & 2,71 \\
Araraquara 2 & 2,64 & 2,83 \\
Campinas & 2,51 & 2,54 \\
\hline
\end{tabular}

Pode ser verificado que o follow-up headway não alterou significativamente em função da faixa utilizada para a entrada na rotatória.

O impacto da escolha do método para a determinação da capacidade de entrada pode ser significativo, portanto é importante validar os resultados obtidos com a finalidade de verificar se há algum método que deve ser evitado. A validação para as rotatórias analisadas foi realizada a partir da comparação das curvas de capacidade para cada método estudado com os dados obtidos em campo em momentos de saturação na entrada da rotatória. Como o fluxo de veículos não é constante, Vasconcelos (2014) sugere realizar análise agregada em dados obtidos a cada minuto, selecionando, aproximadamente, vinte intervalos de um minuto com maior saturação. Para as rotatórias analisadas e utilizando o modelo de capacidade de Hagring (1998), a validação dos dados foi conforme a Figura 1 a seguir. 
Araraquara 1 - Esquerda

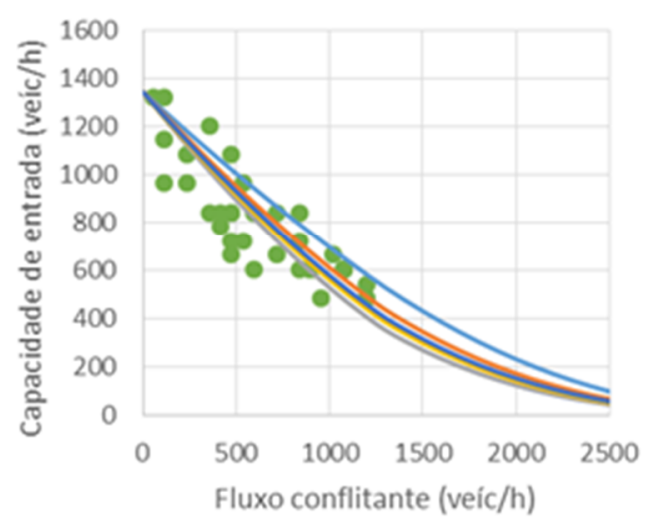

Araraquara 2 - Esquerda

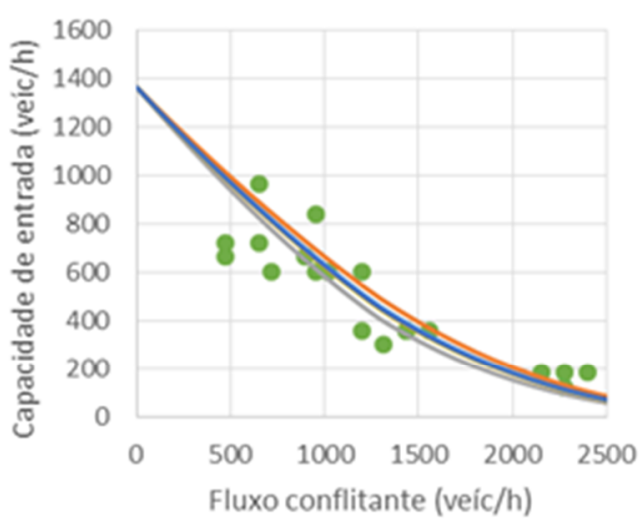

Campinas - Esquerda

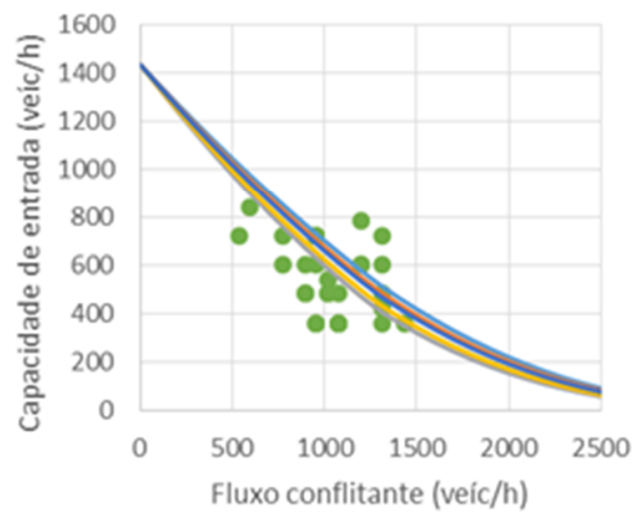

- Dados observados — Siegloch - Raff
Araraquara 1 - Direita

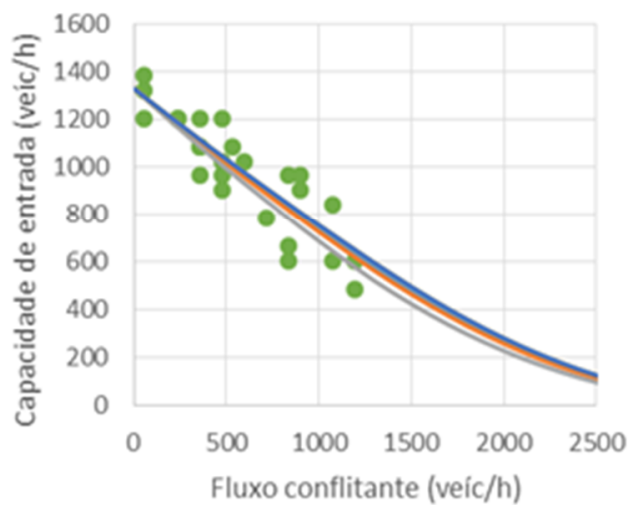

Araraquara 2 - Direita

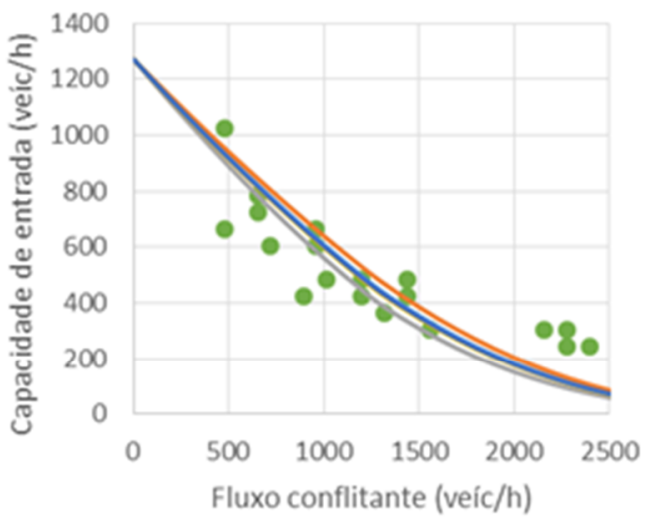

Campinas - Direita

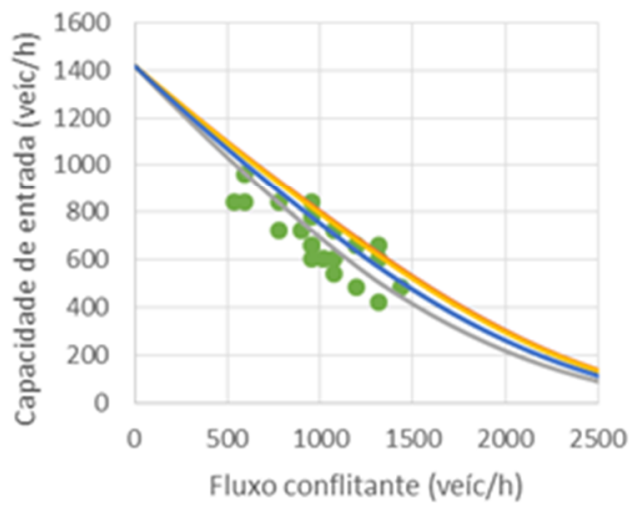

Miller \& Pretty - Wu - Bunker

Figura 1. Validação dos dados de gap crítico e de follow-up headway

Pode-se observar que a variação entre os dados coletados em períodos de saturação é maior que a variação de capacidade obtida pelos métodos de determinação de gap crítico, validando assim os cinco métodos abordados para as interseções em análise.

Na Tabela 3 são apresentados os valores resultantes desta pesquisa, ao realizar uma média ponderada em função do fluxo de veículos, e de outras já realizadas em diversos países para o caso de rotatória com duas faixas. Os resultados são apresentados por faixa, exceto para a Índia devido à falta de limitação de faixas (direita e esquerda). 
Tabela 3 - Valores de gaps críticos para rotatórias em diversos países

\begin{tabular}{|c|c|c|c|c|}
\hline \multirow{2}{*}{ Autor } & \multirow{2}{*}{ País } & \multicolumn{2}{|c|}{ Gap crítico (s) } & \multirow{2}{*}{ Método utilizado } \\
\hline & & Esquerda & Direita & \\
\hline Hagring et al. (2003) & Suécia & 4,36 & 4,08 & $\mathrm{MV}$ \\
\hline Lindenmann (2006) & Suíça & 3,49 & 3,45 & MV e S \\
\hline Rodegerdts et al. (2007) & EUA & 4,50 & 4,20 & MV \\
\hline Gazzarri et al. (2012) & Itália & 3,76 & 3,60 & MV e R \\
\hline Vasconcelos et al (2014) & Portugal & 3,40 & 3,20 & $M V, S, R, E P$ e $B$ \\
\hline Rao et al. (2016) & Índia & \multicolumn{2}{|c|}{1,87} & R e EP \\
\hline Presente pesquisa & Brasil & 3,60 & 3,09 & $M V, S, R, E P$ e $B$ \\
\hline
\end{tabular}

sendo: $\quad$ MV: $\quad$ Método da máxima verossimilhança (Miller \& Pretty)

S: $\quad$ Método de Siegloch

R: $\quad$ Método de Raff

EP: $\quad$ Método do equilíbrio das probabilidades $(\mathrm{Wu})$

B: $\quad$ Método de Bunker

Pode ser observado que o Brasil possui a maior diferença de gap crítico entre a faixa da direita e da esquerda. Para a faixa da direita, no Brasil foi obtido o menor valor de gap crítico ao comparar com os outros países que realizaram a análise segregada. Para a faixa da esquerda, ao comparar com os mesmos países, no Brasil foram obtidos valores superiores à Suíça e Portugal.

Para o follow-up headway, os valores são apresentados na Tabela 4, sendo determinados pelo método de Siegloch ou por média de headways (por observação). Os resultados são apresentados por faixa da aproximação, exceto para a Índia, onde não havia limitação de faixas, e para a Suécia, em que o autor apresentou apenas um valor médio para as duas faixas.

Tabela 4 - Valores de follow-up headway para rotatórias em diversos países

\begin{tabular}{|c|c|c|c|c|}
\hline \multirow{2}{*}{ Autor } & \multirow{2}{*}{ País } & \multicolumn{2}{|c|}{ Follow-up Headway (s) } & \multirow{2}{*}{ Método utilizado } \\
\hline & & Esquerda & Direita & \\
\hline Hagring et al. (2003) & Suécia & \multicolumn{2}{|c|}{2,64} & Observação \\
\hline Lindenmann (2006) & Suíça & 2,73 & 2,55 & Siegloch \\
\hline Rodegerdts et al. (2007) & EUA & 3,40 & 3,10 & Observação \\
\hline Gazzarri et al. (2012) & Itália & 2,65 & 2,64 & Observação \\
\hline Vasconcelos et al. (2014) & Portugal & 2,20 & 2,20 & Siegloch \\
\hline Rao et al. (2016) & Índia & \multicolumn{2}{|c|}{1,15} & Observação \\
\hline Presente pesquisa & Brasil & 2,62 & 2,67 & Siegloch \\
\hline
\end{tabular}

O follow-up headway no Brasil obteve uma característica semelhante entre a faixa da esquerda e da direita, assim como em Portugal e Itália. 0 valor encontrado se aproxima bastante dos outros países, excluindo os EUA, que resultou em valores superiores a $30 \%$ que os valores brasileiros.

\section{CONSIDERAÇÕES FINAIS}

O valor do gap crítico é de difícil determinação e precisa ser validado em campo em situações específicas, como fluxo no limite de capacidade durante todo o período de análise, o que é praticamente impossível se não for realizada uma análise de dados agregados em períodos menores.

0 método da máxima verossimilhança (Miller \& Pretty, 1968) foi o método mais conservador entre os analisados, porém é um dos mais utilizados. As diferenças entre os valores obtidos não foram suficientes a ponto de desvalidar algum método nos casos analisados, já que as análises 
de capacidade pelo modelo de Hagring (1998) não representaram distorções em relação aos dados coletados em campo em períodos de saturação.

A diferença nos valores de gaps críticos para as faixas da direita e esquerda foi de grande proporção, o que pode ser justificado pelo fato de que a maioria dos veículos que chegavam à faixa da direita da aproximação invadia a faixa de retenção para aguardar por um gap na própria via circular, devido à dificuldade de visibilidade, consistindo em uma manobra mais próxima à de mudança de faixa. Nos outros países estudados também foram verificados valores de gaps críticos menores para a faixa da direita, porém com menores proporções ao comparar com os dados brasileiros.

Os valores de follow-up headway foram semelhantes entre as faixas utilizadas. Na rotatória de Campinas foram obtidos valores menores, porém não implica em significativa diferença na capacidade de entrada. O Follow-up headway médio encontrando para representar valores brasileiros foi semelhante aos dados obtidos em países como Suécia, Suíça e Itália.

Os dados obtidos podem variar em outras situações tais como rotatórias de faixa simples, de dimensões menores ou locais com características comportamentais diferenciadas dos motoristas, seja por questão cultural ou pela variação do tráfego, porém são valores que podem ser tomados como base para estimativas de capacidade em projetos brasileiros.

\section{REFERÊNCIAS}

Bunker, J.M. (2012) Novel Methods and the Maximum Likelihood Estimation Technique for Estimating Traffic Critical Gap. Journal of Advanced Transportation, v. 48, ํㅡ 6, p. 542-555. DOI: 10.1002/atr.1204.

Federal Highway Administration (2010) Roundabouts: Na Informational Guide. 2ª Edição. McLean: U. S. Department of Transportation.

Gazzarri, A.; M. T. Martello; Antonio P. e Reginald R. S. (2012) Estimation of Gap Acceptance Parameters for HCM 2010 roundabouts Capacity Model Applications. XVIII Urban Transport: Urban Transport and the Environment in the 21st Century, V. 128, p. 309-319. DOI: 10.2495/UT120271.

Hagring, O. (1998) A further generalization of Tanner's formula, Transportation Research Part B: Methodological, v. 32, no6, p. 423-429. DOI: 10.1016/S0191-2615(98)00010-1

Hagring, O. (2000) Estimation of Critical Gaps in Two Major Streams. Transportation Research Part B: Methodological, v. 34, no 4, p. 293-313. DOI: 10.1016/S0191-2615(99)00026-0

Hagring, O.; N. M. Rouphail, e H. A. Sorensen (2003) Comparison of Capacity Models for Two-Lane Roundabouts. Journal of the Transportation Research Board, v 1852, p. 114-123. DOI: 10.3141/1852-15

Lindenmann, H. P. (2006) Capacity of Small Roundabouts with Two-Lane Entries. Journal of the Transportation Research Board, v. 1988, no 1, p 119-126. DOI: 10.1177/0361198106198800115

Mahesh, S.; A. Ahmad e R. Rastogi (2014) An Approach for the Estimation of Entry Flows on Roundabouts. Transportation Planning and Implementation Methodologies for Developing Countries, v. 17, p. 52-62. DOI: 10.1016/j.trpro.2016.11.060

Miller, A.J. e R. L. Pretty (1968) Overtaking on Two-Lane Rural Roads, 4th Australian Road Research Board Conference, v. 4, nº1, p. 582-594.

Polus, A.; Y. Shiftan e S. Shmueli-Lazar (2005) Evaluation of the Waiting-Time Effect on Critical Gaps at Roundabouts by a Logit Model. European Journal of Transport and Infrastructure Research, v. 5, no 1, p. 1-12.

Raff, M. e J. Hart (1950) A volume warrant to urban stop signs. Eno Foundation for Highway Traffic Control, Saugatuck.

Rao, S.; Y. Krishna; P. Atmakuri e P. Bhuyan (2016) Calibration of Performance of Roundabouts Based on Gap Acceptance Parameters Using Simulation for Indian Scenario. Transportation Planning and Implementation Methodologies for Developing Countries, v. 12.

Rodegerdts, L.; M. Blogg; E. Wemple; E. Myers; M. Kyte; M. Dixon; G. List; A. Flannery; R. Troutbeck; W. Brilon; N. Wu; B. Persaud; C. Lyon; D Harkey e D. Carter (2007b). Roundabouts in the United States. NCHRP report 572, Washington, DC: The National Academy of Sciences.

Siegloch, W. (1973) Die Leistungsermittlung an Knotenpunkten ohne Lichtsignalsteuerung, Strassenbau und Strassenverkehrstechnik v. 154. Federal Ministry of Transport.

TRB (2010) Highway Capacity Manual. Transport Research Board. 6th. Edition. Washington: National Academy of Science.

Vasconcelos, A. L. P. (2014) Análise do Desempenho Operacional de Rotundas: Modelos e Aplicações. Tese (Doutoramento em Engenharia Civil) - Faculdade de Ciências, Universidade de Coimbra, Coimbra.

Wu, N. (2012) Equilibrium of Probabilities for Estimating Distribution Function of Critical Gaps at Unsignalized Intersections. Journal of the Transportation Research Board, v. 2286, p. 49-55. DOI: 10.3141/228606. 